\title{
Partially composite dark matter
}

\author{
Masaki Asano ${ }^{a}$ and Ryuichiro Kitano ${ }^{b, c}$ \\ ${ }^{a}$ Physikalisches Institut and Bethe Center for Theoretical Physics, Universität Bonn, \\ Nussallee 12, D-53115 Bonn, Germany \\ ${ }^{b}$ Theory Center, KEK, \\ 1-1 Oho, Tsukuba, Ibaraki 305-0801, Japan \\ ${ }^{c}$ Department of Particle and Nuclear Physics, \\ The Graduate University for Advanced Studies (Sokendai), \\ 1-1 Oho, Tsukuba, Ibaraki 305-0801, Japan \\ E-mail: masano@th.physik.uni-bonn.de, Ryuichiro.Kitano@kek.jp
}

ABSTRACT: In a class of theories where the Higgs field emerges as a pseudo NambuGoldstone boson, it is often assumed that interactions to generate the top Yukawa coupling provide the Higgs potential as well. Such a scenario generically requires a little cancellation in the leading contribution to the Higgs potential, and the electroweak scale is generated by the balance between the leading and the subleading contributions. We, instead, consider the possibility that the contribution from the dark matter particle balances against that from the top quark. The thermal relic of the new particle explains the abundance of dark matter in a consistent region of the parameter space, and the direct detection is found to be promising.

Keywords: Technicolor and Composite Models, Cosmology of Theories beyond the SM

ARXIV EPRINT: 1406.6374 


\section{Contents}

1 Introduction 1

2 The $\mathrm{SO}(5) / \mathrm{SO}(4)$ model 2

3 Higgs potential from dark matter 4

4 Electroweak symmetry breaking $\quad 7$

5 Dark matter abundance and prospects for direct detection 8

5.1 Relic abundance 8

$\begin{array}{lll}5.2 & \text { Direct detection cross section } & 9\end{array}$

6 Summary 11

\section{Introduction}

The discovery of the Higgs boson [1,2] completes the list of the particles in the Standard Model, but, nevertheless, the origin of the Higgs field and its potential remains as mysteries. The Higgs boson mass, $m_{h}=126 \mathrm{GeV}[3,4]$, which is smaller than its vacuum expectation value (VEV), $v=246 \mathrm{GeV}$, is far from the naive expectation from the analogy of QCD, $m_{h} \sim 4 \pi v$, which naturally leads us to consider the possibility that the Higgs field as a pseudo Nambu-Goldstone boson [5-9].

The hypothesis of the partially composite fermions [10] provides a consistent picture for the scenario of the light Higgs boson as a pseudo Nambu-Goldstone boson. The dynamically broken global symmetry to ensure the massless NG boson is explicitly but weakly broken by couplings between the fermions in the Standard Model and operators in the dynamical sector in addition to the gauge interactions in the Standard Model. The coupling induces mixings between the elementary fermions and composite hadronic states in the dynamical sector, and the Yukawa interactions as well as the Higgs potential are generated through the mixing. The top quark should give the most important contribution to the Higgs potential since its Yukawa coupling is the largest. It has been studied that various types of models can indeed reproduce the $126 \mathrm{GeV}$ Higgs boson while explaining the top quark and the $W$ boson masses. (For a recent review, see ref. [11].)

The TeV scale new physics motivated by the origin of the Higgs boson also provides a natural explanation of dark matter of the Universe as thermal relic of a stable $\mathrm{TeV}$ or weak scale particle. If there is such a particle in the scenario of the pseudo Nambu-Goldstone Higgs, one should also consider the contribution to the Higgs potential from the dark matter particle. Interestingly, such a contribution is somewhat needed to generate a realistic 
potential. The constraints from the electroweak precision measurements prefer to have a little hierarchy between the scale of dynamical symmetry breaking, $f$, and the electroweak $\mathrm{VEV}, v$, so that the Standard Model is realized as a good low energy effective theory. If a single source of the potential is dominated such as from the top quark, the naive expectation of the Higgs VEV is zero or of the order of $\pi f$ due to the periodicity of the Higgs potential. Both are clearly not acceptable. A little hierarchy can be accommodated by assuming a cancellation in the leading contribution so that the subleading one becomes important. The presence of the dark matter particle provides another possibility. The Higgs potential is destabilized at the origin by the contribution from the top quark, and stabilized at a small value $v$ by that from the dark matter particle. Dark matter candidates in the models of dynamical electroweak symmetry breaking have been considered in the literatures; e.g., "technibaryon" [12-16] and "topological dark matter" [17-22]. ${ }^{1}$ Also, a Majorana fermion in the strong dynamics as the dark matter particle has been discussed in ref. [26].

In this paper, we study the contributions to the Higgs potential from the weakly interacting massive particle (WIMP) dark matter. As a concrete example, we consider the $\mathrm{SO}(5) / \mathrm{SO}(4)$ model for the Nambu-Goldstone Higgs field [27], and introduce a gauge singlet Majorana fermion as the dark matter particle which couples to the strong sector in a way that $\mathrm{SO}(5)$ symmetry is explicitly broken. The dark matter generates the Higgs potential of the $\sin ^{2} h / f$ type at the leading order of the coupling. This contribution can balance against the $\cos h / f$ type potential generated from the top quark. We find that in the parameter region where the correct size of the Higgs potential is generated, the dark matter abundance is explained simultaneously through the induced coupling between dark matter and the Higgs field. The predicted spin-independent cross sections for the direct detection experiments are found to be consistent with the current experimental bounds, but are large enough to be covered by the future experiments.

This paper is organized as follows. In the next section, we review the minimal composite Higgs model [27] which we use for the basis of our study. In section 3, we calculate the dark-matter contribution to the Higgs potential, and discuss the consistent parameter region in section 4 . The abundance of dark matter and the possibility of the direct detection are studied in section 5. Section 6 is devoted to summary and discussion.

\section{The $\mathrm{SO}(5) / \mathrm{SO}(4)$ model}

We consider the composite Higgs model associated with the $\mathrm{SO}(5) \rightarrow \mathrm{SO}(4)$ symmetry breaking [27]. The unbroken global symmetry $\mathrm{SO}(4)$ together with $\mathrm{U}(1)_{B-L}$ global symmetry contains $\mathrm{SU}(2)_{L} \times \mathrm{U}(1)_{Y}$ gauge group as a subgroup. The unbroken $\mathrm{SO}(4)$ symmetry ensures the custodial symmetry in the strong sector, and thus there is no severe constraints from the $T$-parameter.

The Nambu-Goldstone field, $\pi(x)$, is introduced as

$$
\xi(x)=e^{i \pi^{a}(x) X^{a}},
$$

\footnotetext{
${ }^{1}$ See also refs. [23-25].
} 
where $X^{a}, a=1, \cdots, 4$, are generators of $\mathrm{SO}(5) / \mathrm{SO}(4)$ in the vector representation, $\mathbf{5}$, of $\mathrm{SO}(5)[28,29]$. The $\xi$ field transforms under $\mathrm{SO}(5)$ symmetry as

$$
\xi \rightarrow \hat{g} \xi \hat{h}^{-1}(\pi, \hat{g}),
$$

where $\hat{g} \in \mathrm{SO}(5)$ and $\hat{h} \in \mathrm{SO}(4)$. We take the basis where unbroken $\mathrm{SO}(4)$ generators are embedded as

$$
S^{\alpha}=\left(\begin{array}{cc}
T^{\alpha} & 0 \\
0 & 0
\end{array}\right), \quad \alpha=1, \cdots, 6 .
$$

Therefore the group element $h$ takes the form of

$$
\hat{h}=\left(\begin{array}{ll}
* & 0 \\
0 & 1
\end{array}\right) .
$$

The Higgs field $\Sigma(x)$ is defined as

$$
\Sigma(x)=\xi(x)\left(\begin{array}{l}
0 \\
0 \\
0 \\
0 \\
1
\end{array}\right)=\frac{\sin (h / f)}{h}\left(\begin{array}{c}
h_{1} \\
h_{2} \\
h_{3} \\
h_{4} \\
h \cot (h / f)
\end{array}\right) .
$$

This field transforms homogeneously as $\Sigma \rightarrow \hat{g} \Sigma$ and the upper four components have the quantum numbers of the Higgs field in the Standard Model, and $h^{2}=h_{1}^{2}+h_{2}^{2}+h_{3}^{2}+h_{4}^{2}$. The electroweak symmetry breaking is described as $\langle h\rangle=\left\langle h_{3}\right\rangle \neq 0$, where $f \sin \langle h / f\rangle=$ $v=246 \mathrm{GeV}$.

In the original minimal composite Higgs model where the top and bottom quarks couple to the operators in the spinorial representation, 4, of $\mathrm{SO}(5)$, the Higgs potential with the following form is generated

$$
V(h) \simeq \alpha_{t} \cos \frac{h}{f}-\beta_{t} \sin ^{2} \frac{h}{f},
$$

where we ignore the small contributions from the SM gauge interactions. The first and the second terms are the leading and sub-leading contributions in terms of the expansion with the coupling constants of the interaction terms between the top quark and the dynamical sector. These couplings break the $\mathrm{SO}(5)$ symmetry explicitly since the top and bottom quarks do not fill the complete multiplet of $\mathrm{SO}(5)$. The Higgs potential is generated though the explicit breaking.

By denoting $\lambda_{q}$ and $\lambda_{u}$, respectively, as the dimensionless couplings of $q=(t, b)$ and $t^{c}$ to the dynamical sector, the naive estimates of $\alpha_{t}$ and $\beta_{t}$ are

$$
\alpha_{t}=\frac{c_{q} \lambda_{q}^{2}+c_{u} \lambda_{u}^{2}}{(4 \pi)^{2}} N_{c} m_{t^{\prime}}^{2} f_{t^{\prime}}^{2}, \quad \beta_{t}=\frac{c_{\beta_{t}} \lambda_{q}^{2} \lambda_{u}^{2}}{(4 \pi)^{2}} N_{c} f_{t^{\prime}}^{4}
$$

where $c_{q}, c_{u}$ and $c_{\beta_{t}}$ are unknown $O(1)$ parameters, and $f_{t^{\prime}}$ and $m_{t^{\prime}}$ are coupling and masses of the lowest resonance to which the operator couples. The top Yukawa coupling is written as

$$
y_{t}=\frac{c_{t} \lambda_{q} \lambda_{u} f_{t^{\prime}}^{2}}{m_{t^{\prime}} f},
$$


with an $O(1)$ parameter, $c_{t}$.

The coefficients $\alpha_{t}$ and $\beta_{t}$ are $O\left(\lambda_{q, u}^{2}\right)$ and $O\left(\lambda_{q, u}^{4}\right)$. On the other hand, from the minimization of the potential, we find

$$
v=246 \mathrm{GeV}=\sqrt{1-\frac{\alpha_{t}^{2}}{4 \beta_{t}^{2}}} \times f .
$$

We need $\alpha_{t}<2 \beta_{t}$ for the vacuum to be stable, which either means that the perturbative expansions in terms of $\lambda$ 's are violated or there is some accidental cancellation in $\alpha_{t}$. Phenomenologically, one needs $v / f \lesssim 0.25$ to satisfy experimental constraints, especially from the $Z b \bar{b}$ coupling [30], which means $\alpha_{t} \simeq 2 \beta_{t}$.

In the following we consider the possibility that $\alpha_{t} \gg \beta_{t}$ as expected from the perturbative expansion, but a large $\sin ^{2}(h / f)$ term necessary for the electroweak symmetry breaking is supplied by the contribution from the dark matter particle.

The models in which SM fermions couple to operators in the fundamental, 5 , or the antisymmetric, 10 representation have also been proposed [31,32] and reported that such models relax the constraints from the $Z b \bar{b}$ coupling, $v / f<0.3-0.4$. For other possibilities see, e.g., ref. [33]. In this paper, we consider the original model in which top and bottom quarks couple to the operators in the spinorial representation, $\mathbf{4}$, since that is the simplest option to incorporate the dark matter particle. By embedding the Standard Model singlet fermion in the $\mathbf{5}$ representation as we explain later, one can generate the potential which can balance against the contributions from the top quark. See also refs. [27, 33-39] for other possible ways for natural electroweak symmetry breaking.

\section{Higgs potential from dark matter}

We introduce the dark matter field, $\psi_{S}$, which is a Majorana fermion and singlet under the SM gauge group. We assume that the dark matter field couples to the dynamical sector as

$$
\mathcal{L} \ni-\frac{m}{2} \bar{\psi}_{S} \psi_{S}+\lambda \bar{\psi}_{S} \mathcal{O}_{5}+i \lambda^{\prime} \bar{\psi}_{S} \gamma_{5} \mathcal{O}_{5}
$$

where $\mathcal{O}_{5}$ is a Majorana fermionic operator in the dynamical sector, and is a component of $\mathrm{SO}(5)$ vector representation,

$$
\mathcal{O}=\left(\begin{array}{c}
\mathcal{O}_{1} \\
\mathcal{O}_{2} \\
\mathcal{O}_{3} \\
\mathcal{O}_{4} \\
\mathcal{O}_{5}
\end{array}\right)
$$

The real valued couplings $\lambda$ and $\lambda^{\prime}$ break the $\mathrm{SO}(5)$ symmetry explicitly. As we will see later, the interaction between the dark matter and the dynamical sector gives a mass to the dark matter which we assume to be the dominant contribution. In that case, one can ignore the mass term $m$ in eq. (3.1), which in turn makes it possible to eliminate the $\lambda^{\prime}$ term by a field redefinition of $\psi_{S}$. 
The mass of $\psi_{S}$ being dominated by the dynamics is simply an assumption. We assume that there is a light fermion in the low energy effective theory. Once we could avoid the Planck scale mass which may arise from quantum gravity, our assumption is in a sense natural since a $\mathrm{U}(1)$ chiral symmetry, $\psi_{S} \rightarrow e^{i \gamma_{5} \theta} \psi_{S}$, is enhanced when we send the $\lambda$ coupling to zero.

The 2-point function of $\psi_{S}$ is written as,

$$
\left\langle\psi_{S}(x) \bar{\psi}_{S}(0)\right\rangle=-\int \frac{d^{4} k}{i(2 \pi)^{4}} \frac{e^{-i k x}}{\not k+\lambda^{2} \Pi_{55}(k)},
$$

where

$$
\begin{aligned}
\Pi_{i j}(q) & =i \int d^{4} x\left\langle\mathcal{O}_{i}(x) \overline{\mathcal{O}}_{j}(0)\right\rangle e^{i q x} \\
& =\Pi_{4}(q)\left(\delta_{i j}-\Sigma_{i} \Sigma_{j}\right)+\Pi_{1}(q) \Sigma_{i} \Sigma_{j} .
\end{aligned}
$$

In the last expression of eq. (3.4), we decompose ח's in terms of the unbroken $\mathrm{SO}(4)$ symmetry. The field $\Sigma$ is treated as an external field. The $\Pi_{4}$ and $\Pi_{1}$ functions can be expressed in terms of the spectral functions such as

$$
\begin{aligned}
& \Pi_{4}(q)=-\int_{0}^{\infty} d s \frac{q \rho_{4}(s)+\tilde{\rho}_{4}(s)+i \gamma_{5} \tilde{\rho}_{4,5}(s)}{q^{2}-s+i \epsilon}+\cdots \\
& \Pi_{1}(q)=-\int_{0}^{\infty} d s \frac{q \rho_{1}(s)+\tilde{\rho}_{1}(s)+i \gamma_{5} \tilde{\rho}_{1,5}(s)}{q^{2}-s+i \epsilon}+\cdots
\end{aligned}
$$

where the ellipsis are regular functions of $q^{2}$, representing the contact terms. ${ }^{2}$

In the case where there is an effective description in terms of weakly coupled composite states, such as in a large $N$ theory, spectral functions are approximated as collections of hadron poles:

$$
\begin{aligned}
\rho_{4}(s) & =\sum_{i} f_{4, i}^{2} \delta\left(s-\left|m_{4, i}\right|^{2}\right), & \rho_{1}(s) & =\sum_{i} f_{1, i}^{2} \delta\left(s-\left|m_{1, i}\right|^{2}\right), \\
\tilde{\rho}_{4}(s) & =\sum_{i} f_{4, i}^{2} \operatorname{Re}\left[m_{4, i}\right] \delta\left(s-\left|m_{4, i}\right|^{2}\right), & \tilde{\rho}_{1}(s) & =\sum_{i} f_{1, i}^{2} \operatorname{Re}\left[m_{1, i}\right] \delta\left(s-\left|m_{1, i}\right|^{2}\right), \\
\tilde{\rho}_{4,5}(s) & =\sum_{i} f_{4, i}^{2} \operatorname{Im}\left[m_{4, i}\right] \delta\left(s-\left|m_{4, i}\right|^{2}\right), & \tilde{\rho}_{1,5}(s) & =\sum_{i} f_{1, i}^{2} \operatorname{Im}\left[m_{1, i}\right] \delta\left(s-\left|m_{1, i}\right|^{2}\right) .
\end{aligned}
$$

We assume that $\mathrm{SO}(5)$ symmetry is broken by a VEV of some composite operator, $X$, with the mass dimension $d$. Then it contributes to $\Pi_{4}(q)-\Pi_{1}(q)$ as $\propto\left\langle X^{\dagger} X\right\rangle / q^{2 d-1}$ for a large $q$. This condition gives the Weinberg sum rules for the spectral functions:

$$
\int_{0}^{\infty} d s\left(\rho_{4}(s)-\rho_{1}(s)\right)=0, \quad(d>1)
$$

\footnotetext{
${ }^{2}$ The two point functions of Majorana operators satisfy

$$
\Pi(q)=-C \Pi^{\mathrm{T}}(-q) C, \quad \Pi^{\dagger}(q)=\gamma^{0} \Pi(q) \gamma^{0},
$$
}

which forbid the terms proportional to $\gamma_{5} q$. 


$$
\begin{aligned}
& \int_{0}^{\infty} d s\left(\tilde{\rho}_{4}(s)-\tilde{\rho}_{1}(s)\right)=0, \quad \int_{0}^{\infty} d s\left(\tilde{\rho}_{4,5}(s)-\tilde{\rho}_{1,5}(s)\right)=0, \quad(d>3 / 2), \\
& \int_{0}^{\infty} d s \cdot s\left(\rho_{4}(s)-\rho_{1}(s)\right)=0, \quad(d>2), \\
& \int_{0}^{\infty} d s \cdot s\left(\tilde{\rho}_{4}(s)-\tilde{\rho}_{1}(s)\right)=0, \quad \int_{0}^{\infty} d s \cdot s\left(\tilde{\rho}_{4,5}(s)-\tilde{\rho}_{1,5}(s)\right)=0, \quad(d>5 / 2) .
\end{aligned}
$$

For example, if $X$ is a fermion pair in an asymptotically free theory, $d=3$, and the above six sum rules apply. One can also obtain a relation that the contact terms in eqs. (3.5) and (3.6) are common for $\Pi_{4}(q)$ and $\Pi_{1}(q)$.

The Higgs potential can be calculated by using the Coleman-Weinberg formula:

$$
\begin{aligned}
V(h) & =-\frac{1}{2} \int \frac{d^{4} k}{i(2 \pi)^{4}} \operatorname{Tr} \log \left[\not k+\lambda^{2} \Pi_{55}(k)+i \epsilon\right] \\
& =\text { const. }-\frac{1}{2} \int \frac{d^{4} k}{i(2 \pi)^{4}} \operatorname{Tr}\left[\frac{-\lambda^{2}}{\not k+i \epsilon}\left(\Pi_{4}(k)-\Pi_{1}(k)\right) \Sigma_{5} \Sigma_{5}\right]+O\left(\lambda^{4}\right) \\
& \equiv \text { const. }-\beta \sin ^{2} \frac{h}{f}+O\left(\lambda^{4}\right),
\end{aligned}
$$

where

$$
\beta=-\frac{1}{2} \cdot \lambda^{2} \int_{0}^{\infty} d s \int \frac{d^{4} k}{i(2 \pi)^{4}} \frac{4 k^{2}\left(\rho_{4}(s)-\rho_{1}(s)\right)}{\left(k^{2}+i \epsilon\right)\left(k^{2}-s+i \epsilon\right)} .
$$

The Weinberg sum rules make the momentum integral converge. The piece which is nonvanishing under the Weinberg sum rules (3.10) and (3.12) is

$$
\beta=\frac{1}{2} \frac{4 \lambda^{2}}{(4 \pi)^{2}} \int_{0}^{\infty} d s \cdot s\left(\rho_{4}(s)-\rho_{1}(s)\right) \log \frac{s}{s_{0}},
$$

where $s_{0}$ is an arbitrary number. The $s_{0}$ independence is ensured by eq. (3.12).

When we set $s_{0}$ as the mass squared of the lowest resonance to couple the operator $\mathcal{O}_{i}, m_{\mathcal{O}}$

$$
\begin{aligned}
\beta & =\frac{1}{2} \frac{4 \lambda^{2}}{(4 \pi)^{2}} \int_{m_{\mathcal{O}}^{2}}^{\infty} d s \cdot s\left(\rho_{4}(s)-\rho_{1}(s)\right) \log \frac{s}{m_{\mathcal{O}}^{2}} \\
& =-\frac{1}{2} \frac{4 \lambda^{2}}{(4 \pi)^{2}} \int_{m_{\mathcal{O}}^{2}}^{\infty} d s \cdot \Delta(s)\left(1+\log \frac{s}{m_{\mathcal{O}}^{2}}\right)
\end{aligned}
$$

where

$$
\Delta(s)=\int_{m_{\mathcal{O}}^{2}}^{s} d s^{\prime}\left(\rho_{4}\left(s^{\prime}\right)-\rho_{1}\left(s^{\prime}\right)\right)
$$

The function $\Delta(s)$ goes to zero as $s \rightarrow \infty$ because of eq. (3.10). Therefore, the integration in eq. (3.17) should be dominated by the lower resonances. Therefore, we expect

$$
\beta=\frac{1}{2} \frac{4 \lambda^{2}}{(4 \pi)^{2}} c_{\beta} m_{\mathcal{O}}^{2} f_{\mathcal{O}}^{2},
$$

where $c_{\beta}$ is an $O(1)$ coefficient, and $f_{\mathcal{O}}$ is the coupling of the lowest resonance which couples to the operator $\mathcal{O}_{i}$. The overall sign depends on that of $\rho_{4}(s)-\rho_{1}(s)$ near $s \sim m_{\mathcal{O}}^{2}$. We assume $\beta>0$ which is necessary for the vacuum to be stable. 


\section{Electroweak symmetry breaking}

Adding the contribution in eq. (3.14) to eq. (2.6), the total Higgs potential is obtained as

$$
V(h)=\alpha_{t} \cos \frac{h}{f}-\left(\beta+\beta_{t}\right) \sin ^{2} \frac{h}{f} .
$$

The minimization of the potential gives the electroweak VEV and the Higgs mass as follows:

$$
\begin{gathered}
v=246 \mathrm{GeV}=\sqrt{1-\frac{\alpha_{t}^{2}}{4\left(\beta+\beta_{t}\right)^{2}}} \times f \equiv \epsilon f, \\
m_{h}^{2}=(126 \mathrm{GeV})^{2}=\frac{2\left(\beta+\beta_{t}\right) \epsilon^{2}}{f^{2}} .
\end{gathered}
$$

Therefore, in the case where the dark matter contribution exists, the stable minimum can be found for $\alpha_{t} \gg \beta_{t}$. A small $\epsilon$ can be obtained when $\beta \simeq \alpha_{t} \gg \beta_{t}$. When $\beta_{t}$ is negligible, from eq. (3.19), we find

$$
m_{h}^{2}=c_{\beta} \cdot \epsilon^{2} \cdot \frac{4 \lambda^{2}}{(4 \pi)^{2}} m_{\mathcal{O}}^{2}\left(\frac{f_{\mathcal{O}}}{f}\right)^{2}
$$

From this, we obtain the mass of the first resonance to be

$$
m_{\mathcal{O}}=4.9 \mathrm{TeV} \cdot c_{\beta}^{-1 / 2}\left(\frac{\lambda f_{\mathcal{O}}}{1 \mathrm{TeV}}\right)^{-1}\left(\frac{\epsilon}{0.2}\right)^{-2} .
$$

On the other hand, from eq. (4.2), $\alpha_{t}$ is required to satisfy:

$$
\alpha_{t}=2\left(\beta+\beta_{t}\right) \sqrt{1-\epsilon^{2}} \simeq 2 \beta=\frac{m_{h}^{2} v^{2}}{\epsilon^{4}} .
$$

The mass of the lowest top partner resonance is, therefore, given by

$$
m_{t^{\prime}}=2.4 \mathrm{TeV}\left(\frac{c_{t} \cdot 2 \lambda_{q} \lambda_{u}}{c_{q} \lambda_{q}^{2}+c_{u} \lambda_{u}^{2}}\right)^{1 / 3}\left(\frac{\epsilon}{0.2}\right)^{-1} \leq 2.4 \mathrm{TeV}\left(\frac{c_{t}}{\sqrt{c_{q} c_{u}}}\right)^{1 / 3}\left(\frac{\epsilon}{0.2}\right)^{-1} .
$$

Here, we have used $m_{h}=126 \mathrm{GeV}$ and $m_{t}=173 \mathrm{GeV}$. The correct top quark mass requires

$$
\frac{\lambda_{q} \lambda_{u} f_{t^{\prime}}^{2}}{m_{t^{\prime}}^{2}}=0.5 \cdot c_{t}^{-1}\left(\frac{\epsilon}{0.2}\right)^{-1}\left(\frac{m_{t^{\prime}}}{2.4 \mathrm{TeV}}\right)^{-1} .
$$

The assumption that perturbative expansions by $\lambda, \lambda_{q}$ and $\lambda_{u}$ make sense requires

$$
\frac{\lambda^{2} f_{\mathcal{O}}^{2}}{m_{\mathcal{O}}^{2}}<1, \quad \frac{\lambda_{q, u}^{2} f_{t^{\prime}}^{2}}{m_{t^{\prime}}^{2}}<1
$$

Compared with eq. (4.8), we need somewhat large $c_{t}$ and/or $m_{t^{\prime}}$ for reliable perturbative estimates while explaining the top quark mass. We assume that is the case since otherwise the whole estimation as well as the assumption of the small $\beta_{t}$ become unreliable. We also expect that $m_{\mathcal{O}} \sim m_{t^{\prime}}$ since they are both hadrons in the same dynamics. Putting altogether, we find $\lambda f_{\mathcal{O}} \sim 1-2 \mathrm{TeV}$ and $m_{\mathcal{O}} \sim m_{t^{\prime}} \sim 2-4 \mathrm{TeV}$ provides the successful electroweak symmetry breaking within the perturbative regime. We will see below that the correct abundance of dark matter is obtained in the same parameter region. 


\section{Dark matter abundance and prospects for direct detection}

By integrating out the dynamical sector, the mass and coupling of the dark matter particle are generated such as

$$
\begin{aligned}
\mathcal{L}_{\mathrm{eff}}= & -\frac{m_{\mathrm{DM}}}{2} \bar{\psi}_{S} \psi_{S}-i \frac{m_{\mathrm{DM}, 5}}{2} \bar{\psi}_{S} \gamma_{5} \psi_{S} \\
& +\frac{\kappa}{2} \bar{\psi}_{S} \psi_{S} \sin ^{2} \frac{h}{f}+\frac{i \kappa_{5}}{2} \bar{\psi}_{S} \gamma_{5} \psi_{S} \sin ^{2} \frac{h}{f} .
\end{aligned}
$$

At the leading order in $\lambda$ and $\epsilon$, they are given by

$$
\begin{aligned}
m_{\mathrm{DM}} & =-\lambda^{2} \int_{0}^{\infty} d s \frac{\tilde{\rho}_{1}(s)}{s}, & m_{\mathrm{DM}, 5} & =-\lambda^{2} \int_{0}^{\infty} d s \frac{\tilde{\rho}_{1,5}(s)}{s}, \\
\kappa & =\lambda^{2} \int_{0}^{\infty} d s \frac{\tilde{\rho}_{4}(s)-\tilde{\rho}_{1}(s)}{s}, & \kappa_{5} & =\lambda^{2} \int_{0}^{\infty} d s \frac{\tilde{\rho}_{4,5}(s)-\tilde{\rho}_{1,5}(s)}{s} .
\end{aligned}
$$

The couplings to the gauge bosons are suppressed by $\epsilon^{2}$. One can eliminate the $m_{\mathrm{DM}, 5}$ term by the redefinition of $\psi_{S}$. In that basis, $m_{\mathrm{DM}}, \kappa$ and $\kappa_{5}$ are shifted to

$$
m_{\mathrm{DM}} \rightarrow \sqrt{m_{\mathrm{DM}}^{2}+m_{\mathrm{DM}, 5}^{2}}, \quad \kappa \rightarrow \kappa \cos \tilde{\theta}+\kappa_{5} \sin \tilde{\theta}, \quad \kappa_{5} \rightarrow \kappa \sin \tilde{\theta}+\kappa_{5} \cos \tilde{\theta},
$$

where $\tan \tilde{\theta}=m_{\mathrm{DM}} / m_{\mathrm{DM}, 5}$. In general, $\kappa_{5}$ cannot be eliminated simultaneously. Since we expect from the dimensional analysis that the dark matter mass and couplings are $O\left(\lambda^{2} f_{\mathcal{O}}^{2} / m_{\mathcal{O}}\right)$, we take the parameters in eq. (5.1) as

$$
m_{\mathrm{DM}}=c_{\mathrm{DM}} \frac{\lambda^{2} f_{\mathcal{O}}^{2}}{m_{\mathcal{O}}}, \quad m_{\mathrm{DM}, 5}=0, \quad \kappa=c_{\kappa} \frac{\lambda^{2} f_{\mathcal{O}}^{2}}{m_{\mathcal{O}}}, \quad \kappa_{5}=c_{\kappa_{5}} \frac{\lambda^{2} f_{\mathcal{O}}^{2}}{m_{\mathcal{O}}},
$$

with $O(1)$ parameters, $c_{\mathrm{DM}}, c_{\kappa}$ and $c_{\kappa_{5}} \cdot{ }^{3}$

\subsection{Relic abundance}

Since we expect $\kappa_{5} \sim \kappa$, the annihilation via the $\kappa_{5}$ coupling mainly contributes to determine the relic density of dark matter because it is an $s$-wave process whereas the one with $\kappa$ is $p$-wave. The annihilation cross section is given by

$$
\begin{aligned}
\left\langle\sigma_{\mathrm{ann} .} v\right\rangle \simeq & 4 s\left(\frac{\kappa_{5}}{f^{2}}\right)^{2} \frac{v^{2}}{\left(s-m_{h}^{2}\right)^{2}+m_{h}^{2} \Gamma_{h}^{2}} \frac{\left.\Gamma_{h}\right|_{m_{h}=\sqrt{s}}}{\sqrt{s}} \\
& +\frac{1}{8 \pi}\left(\frac{\kappa_{5}}{f^{2}}\right)^{2}\left(1+\frac{3 m_{h}^{2}}{s-m_{h}^{2}}\right)^{2}\left(1-\frac{4 m_{h}^{2}}{s}\right)^{1 / 2}
\end{aligned}
$$

for $m_{\mathrm{DM}}>m_{h}$, and $s$ and $\Gamma_{h}$ are the center of mass energy $\left(\sim 2 m_{\mathrm{DM}}\right)$ and the total decay width of the Higgs boson, respectively. In the limit of heavy dark matter, $m_{\mathrm{DM}} \gg m_{h}$, it simplifies to

$$
\left\langle\sigma_{\mathrm{ann} .} v\right\rangle \simeq \frac{1}{2 \pi}\left(\frac{\kappa_{5}}{f^{2}}\right)^{2}
$$

\footnotetext{
${ }^{3}$ Phenomenology of dark matter candidates which have a similar effective interactions has been studied in refs. [40-43].
} 


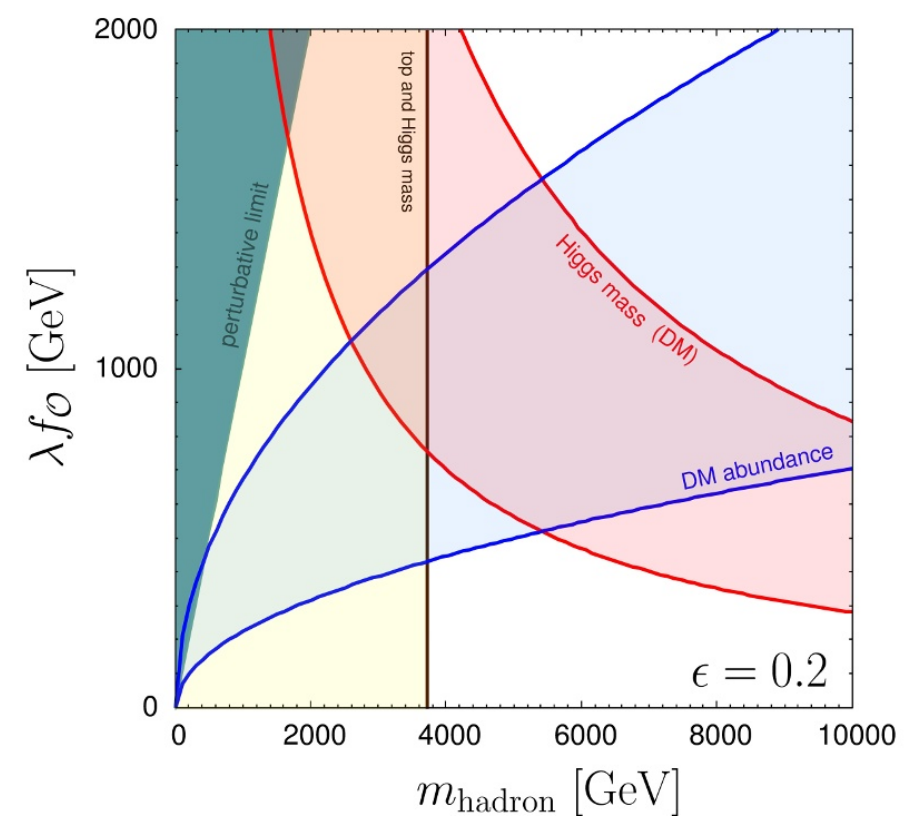

Figure 1. Consistent parameter regions are shown for $\epsilon=0.2$. The bands are drawn by taking $O(1)$ parameters to range $1 / 3<c_{X}<3$.

The dependence on the $m_{\mathrm{DM}}$ disappears.

Requiring $\Omega_{\mathrm{DM}} h^{2}=0.12$ [44], we find

$$
\kappa_{5}=160 \mathrm{GeV}\left(\frac{\epsilon}{0.2}\right)^{-2}
$$

which means

$$
\lambda f_{\mathcal{O}}=900 \mathrm{GeV} \cdot c_{\kappa_{5}}^{-1 / 2}\left(\frac{m_{\mathcal{O}}}{5 \mathrm{TeV}}\right)^{1 / 2}\left(\frac{\epsilon}{0.2}\right)^{-1},
$$

from eq. (5.5). Barring $O(1)$ uncertainties in various estimates, the value is comfortably consistent with the requirements from the Higgs and top quark masses. We summarize the consistent parameter regions in figure 1 where we allow $O(1)$ parameters to range $1 / 3<c<3$. The requirements from the top quark mass, the Higgs boson mass, the dark matter abundance all agree in the region where the perturbative expansion is reliable.

\subsection{Direct detection cross section}

From eqs. (5.5) and (5.8), the dark matter mass is obtained as

$$
m_{\mathrm{DM}}=160 \mathrm{GeV}\left(\frac{c_{\mathrm{DM}}}{c_{\kappa_{5}}}\right)\left(\frac{\epsilon}{0.2}\right)^{-2} .
$$

The on-going direct detection experiments have good sensitivity for such a weak scale dark matter.

In contrast to the case of the annihilation process, the $\kappa$ coupling, rather than $\kappa_{5}$, mainly contributes to the scattering processes for the direct detection since the $\kappa_{5}$ coupling only contributes to the spin-dependent part in the non-relativistic limit. The spin 


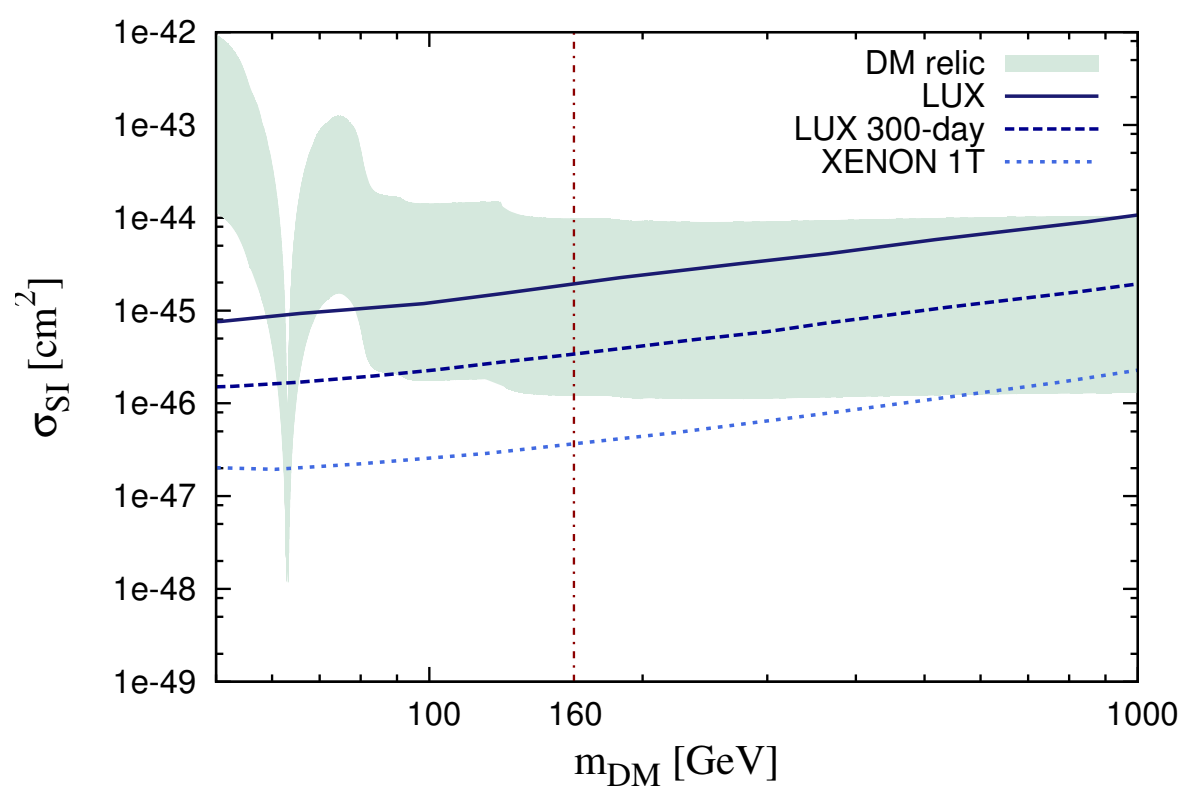

Figure 2. Spin independent cross section of the dark matter-nucleon scattering.

independent cross section per nucleon is given by

$$
\begin{aligned}
\sigma_{\mathrm{SI}} & =\frac{4}{\pi}\left(\frac{m_{N} m_{\mathrm{DM}}}{m_{N}+m_{\mathrm{DM}}}\right)^{2} \frac{\left[Z f_{p}+(A-Z) f_{n}\right]^{2}}{A^{2}}, \\
f_{N} & =\frac{\kappa}{f^{2}} \frac{m_{N}}{m_{h}^{2}}\left[\sum_{q=u, d, s} f_{q}^{N}+\frac{2}{9}\left(1-\sum_{q=u, d, s} f_{q}^{N}\right)\right],
\end{aligned}
$$

where $m_{N}(N=p, n)$ is the nucleon mass and $A$ and $Z$ are the mass and atomic number of the target nucleus, respectively. The factor $f_{q}^{N}$ are matrix elements, $f_{q}^{N}=$ $\left(m_{q} / m_{N}\right)\langle N|\bar{q} q| N\rangle$. Assuming $f_{q}^{n}=f_{q}^{p}$ and taking the following values, $f_{u}^{p}=0.021$, $f_{d}^{p}=0.029$ and $f_{s}^{p}=0.009[45,46],{ }^{4}$ which provide us with conservative estimates, the cross section is given by

$$
\sigma_{\mathrm{SI}} \simeq 1.2 \times 10^{-45} \mathrm{~cm}^{2}\left(\frac{c_{\kappa}}{c_{\kappa 5}}\right)^{2},
$$

where we use the information of the annihilation cross section in eq. (5.8).

Figure 2 shows the spin independent cross section of the dark matter scattering on proton. In the shaded region, the thermal dark matter abundance is consistent with the current observation and, in this figure, we assume $1 / 3<c_{\kappa} / c_{\kappa_{5}}<3$. The solid line and dashed line show the upper bound on the cross section from the LUX experiment and the expected upper bound from future LUX 300-day run, respectively $[49,50]$. The dotted line denotes the expected upper bound from the future XENON 1T experiment [51]. ${ }^{5}$ As one can see, a large parameter region can be covered by direct detection experiments in near future.

\footnotetext{
${ }^{4}$ If we take other values in the literatures, e.g., in refs. [47, 48], the cross section changes by $O(10 \%)$.

${ }^{5}$ The constraints from indirect detections of dark matter turn out to be not quite strong [52].
} 
Here, we comment on other possible signatures of the model. The compositeness of the Higgs boson affects the coupling of the Higgs boson. In particular, the coupling to electroweak gauge bosons can be measured with a good accuracy. It has been studied that the sensitivity can reach to $\epsilon \sim 0.1(0.01)$ at the LHC (ILC) [11]. ${ }^{6}$ In the $m_{\mathrm{DM}}<m_{h} / 2$ region, the invisible branching ratio of the Higgs boson decay is also expected. The current searches at the LHC [54-57] put an upper bound around 20\% [58] from the global fit assuming that the coupling constants are not modified from the Standard Model ones.

Multi-TeV spin-1/2 resonances are expected in the top quark and dark matter sector as discussed above. The top partner with the same charge of top quark may be accessible at future collider experiments $\left(<3.2 \mathrm{TeV}\right.$ by LHC $33 \mathrm{TeV}$ with $\left.3 \mathrm{ab}^{-1}[59,60]\right)$.

\section{Summary}

Dark matter of the Universe and the Higgs boson are two mysterious items in particle physics, and probably hints for deeper understanding of particle physics are hidden there. Indeed, the size of the interaction required to explain the abundance of dark matter by the thermal relic is of the order of the weak interaction, that is characterized by the Higgs VEV. This may be telling us that the nature of dark matter and that of the Higgs boson are tightly related.

We consider the possibility that dark matter is the one which is responsible for creating the potential of the Higgs field. We see that in the minimal composite Higgs model, the balance between the potentials made by the top quark and the dark matter can trigger the successful electroweak symmetry breaking while explaining the abundance of the dark matter.

We have studied the effective theory where the Higgs field is described as the pseudo Nambu-Goldstone boson. In the language of the effective theory, the Higgs field is already introduced as effective degrees of freedom, and we assume that the dark matter particle couples to it through some interaction term to break the global symmetry. In a full dynamical description, however, the picture may be more dramatic; the Higgs field may actually be the condensation of dark matter. For example, it has been studied recently that the scenario of the top quark condensation can have a picture of the pseudo Nambu-Goldstone Higgs boson [61, 62]. It is promising that the realization of the dark-matter condensation as the Higgs field is also possible.

In the parameter region where the Higgs boson mass and the abundance of the dark matter is explained, the spin-independent cross section for the direct detection experiments turns out to be quite large, just below the experimental constraints. If there is no significant fine-tuning, we expect to see the detection quite soon.

\section{Acknowledgments}

This work is supported by JSPS Grant-in-Aid for Young Scientists (B) (No. 23740165 [RK]), MEXT Grant-in-Aid for Scientific Research on Innovative Areas (No. 25105011 $[\mathrm{RK}]$ ) and the German Research Foundation through TRR33 "The Dark Universe" (MA).

\footnotetext{
${ }^{6}$ For details, see also ref. [53].
} 
Open Access. This article is distributed under the terms of the Creative Commons Attribution License (CC-BY 4.0), which permits any use, distribution and reproduction in any medium, provided the original author(s) and source are credited.

\section{References}

[1] ATLAS collaboration, Observation of a new particle in the search for the Standard Model Higgs boson with the ATLAS detector at the LHC, Phys. Lett. B 716 (2012) 1 [arXiv: 1207.7214] [INSPIRE].

[2] CMS collaboration, Observation of a new boson at a mass of $125 \mathrm{GeV}$ with the CMS experiment at the LHC, Phys. Lett. B 716 (2012) 30 [arXiv:1207.7235] [INSPIRE].

[3] ATLAS collaboration, Measurements of the properties of the Higgs-like boson in the two photon decay channel with the ATLAS detector using $25 \mathrm{fb}^{-1}$ of proton-proton collision data, ATLAS-CONF-2013-012, CERN, Geneva Switzerland (2013).

[4] CMS collaboration, Updated measurements of the Higgs boson at $125 \mathrm{GeV}$ in the two photon decay channel, CMS-PAS-HIG-13-001, CERN, Geneva Switzerland (2013).

[5] D.B. Kaplan and H. Georgi, $\mathrm{SU}(2) \times \mathrm{U}(1)$ breaking by vacuum misalignment, Phys. Lett. B 136 (1984) 183 [INSPIRE].

[6] D.B. Kaplan, H. Georgi and S. Dimopoulos, Composite Higgs scalars, Phys. Lett. B 136 (1984) 187 [INSPIRE].

[7] H. Georgi, D.B. Kaplan and P. Galison, Calculation of the composite Higgs mass, Phys. Lett. B 143 (1984) 152 [INSPIRE].

[8] H. Georgi and D.B. Kaplan, Composite Higgs and custodial SU(2), Phys. Lett. B 145 (1984) 216 [INSPIRE].

[9] M.J. Dugan, H. Georgi and D.B. Kaplan, Anatomy of a composite Higgs model, Nucl. Phys. B 254 (1985) 299 [INSPIRE].

[10] D.B. Kaplan, Flavor at SSC energies: a new mechanism for dynamically generated fermion masses, Nucl. Phys. B 365 (1991) 259 [InSPIRE].

[11] B. Bellazzini, C. Csáki and J. Serra, Composite Higgses, Eur. Phys. J. C 74 (2014) 2766 [arXiv: 1401.2457] [INSPIRE].

[12] S. Nussinov, Technocosmology: could a technibaryon excess provide a 'natural' missing mass candidate?, Phys. Lett. B 165 (1985) 55 [INSPIRE].

[13] S.M. Barr, R.S. Chivukula and E. Farhi, Electroweak fermion number violation and the production of stable particles in the early universe, Phys. Lett. B 241 (1990) 387 [INSPIRE].

[14] S.B. Gudnason, C. Kouvaris and F. Sannino, Towards working technicolor: effective theories and dark matter, Phys. Rev. D 73 (2006) 115003 [hep-ph/0603014] [INSPIRE].

[15] S.B. Gudnason, C. Kouvaris and F. Sannino, Dark matter from new technicolor theories, Phys. Rev. D 74 (2006) 095008 [hep-ph/0608055] [INSPIRE].

[16] R. Foadi, M.T. Frandsen and F. Sannino, Technicolor dark matter, Phys. Rev. D 80 (2009) 037702 [arXiv: 0812.3406] [INSPIRE].

[17] H. Murayama and J. Shu, Topological dark matter, Phys. Lett. B 686 (2010) 162 [arXiv: 0905.1720] [INSPIRE]. 
[18] A. Joseph and S.G. Rajeev, Topological dark matter in the little Higgs models, Phys. Rev. D 80 (2009) 074009 [arXiv:0905.2772] [INSPIRE].

[19] M. Gillioz, A. von Manteuffel, P. Schwaller and D. Wyler, The little skyrmion: new dark matter for little Higgs models, JHEP 03 (2011) 048 [arXiv: 1012.5288] [INSPIRE].

[20] M. Gillioz, Dangerous skyrmions in little Higgs models, JHEP 02 (2012) 121 [Erratum ibid. 03 (2013) 123] [arXiv: 1111.2047] [INSPIRE].

[21] J. Ellis, M. Karliner and M. Praszalowicz, Generalized skyrmions in $Q C D$ and the electroweak sector, JHEP 03 (2013) 163 [arXiv:1209.6430] [INSPIRE].

[22] A. Pomarol and A. Wulzer, Stable skyrmions from extra dimensions, JHEP 03 (2008) 051 [arXiv:0712.3276] [INSPIRE].

[23] D. Poland and J. Thaler, The dark top, JHEP 11 (2008) 083 [arXiv:0808.1290] [InSPIRE].

[24] M. Frigerio, A. Pomarol, F. Riva and A. Urbano, Composite scalar dark matter, JHEP 07 (2012) 015 [arXiv: 1204.2808] [INSPIRE].

[25] D. Marzocca and A. Urbano, Composite dark matter and LHC interplay, JHEP 07 (2014) 107 [arXiv: 1404.7419] [INSPIRE].

[26] C. Kouvaris, Dark Majorana particles from the minimal walking technicolor, Phys. Rev. D 76 (2007) 015011 [hep-ph/0703266] [INSPIRE].

[27] K. Agashe, R. Contino and A. Pomarol, The minimal composite Higgs model, Nucl. Phys. B 719 (2005) 165 [hep-ph/0412089] [INSPIRE].

[28] S.R. Coleman, J. Wess and B. Zumino, Structure of phenomenological Lagrangians. 1, Phys. Rev. 177 (1969) 2239 [INSPIRE].

[29] C.G. Callan Jr., S.R. Coleman, J. Wess and B. Zumino, Structure of phenomenological Lagrangians. 2, Phys. Rev. 177 (1969) 2247 [INSPIRE].

[30] K. Agashe and R. Contino, The minimal composite Higgs model and electroweak precision tests, Nucl. Phys. B 742 (2006) 59 [hep-ph/0510164] [INSPIRE].

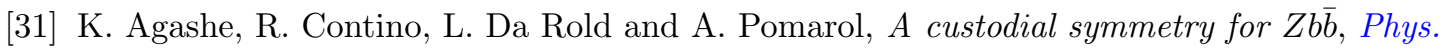
Lett. B 641 (2006) 62 [hep-ph/0605341] [INSPIRE].

[32] R. Contino, L. Da Rold and A. Pomarol, Light custodians in natural composite Higgs models, Phys. Rev. D 75 (2007) 055014 [hep-ph/0612048] [InSPIRE].

[33] A. Pomarol and F. Riva, The composite Higgs and light resonance connection, JHEP 08 (2012) 135 [arXiv:1205.6434] [INSPIRE].

[34] O. Matsedonskyi, G. Panico and A. Wulzer, Light top partners for a light composite Higgs, JHEP 01 (2013) 164 [arXiv: 1204.6333] [INSPIRE].

[35] M. Redi and A. Tesi, Implications of a light Higgs in composite models, JHEP 10 (2012) 166 [arXiv: 1205.0232] [INSPIRE].

[36] D. Marzocca, M. Serone and J. Shu, General composite Higgs models, JHEP 08 (2012) 013 [arXiv: 1205.0770] [INSPIRE].

[37] G. Panico, M. Redi, A. Tesi and A. Wulzer, On the tuning and the mass of the composite Higgs, JHEP 03 (2013) 051 [arXiv:1210.7114] [INSPIRE].

[38] D. Pappadopulo, A. Thamm and R. Torre, A minimally tuned composite Higgs model from an extra dimension, JHEP 07 (2013) 058 [arXiv: 1303.3062] [INSPIRE]. 
[39] L. Vecchi, The natural composite Higgs, arXiv:1304.4579 [INSPIRE].

[40] L. Lopez-Honorez, T. Schwetz and J. Zupan, Higgs portal, fermionic dark matter and a Standard Model like Higgs at 125 GeV, Phys. Lett. B 716 (2012) 179 [arXiv:1203.2064] [INSPIRE].

[41] L. Vecchi, A heavy "neutralino" in warped extra dimensions, Phys. Rev. D 90 (2014) 025017 [arXiv: 1310.7862] [INSPIRE].

[42] A. De Simone, G.F. Giudice and A. Strumia, Benchmarks for dark matter searches at the LHC, JHEP 06 (2014) 081 [arXiv: 1402.6287] [INSPIRE].

[43] M.A. Fedderke, J.-Y. Chen, E.W. Kolb and L.-T. Wang, The fermionic dark matter Higgs portal: an effective field theory approach, JHEP 08 (2014) 122 [arXiv:1404.2283] [INSPIRE].

[44] Planck collaboration, P.A.R. Ade et al., Planck 2013 results. XVI. Cosmological parameters, Astron. Astrophys. (2014) [arXiv:1303.5076] [INSPIRE].

[45] P. Agrawal, Z. Chacko, C. Kilic and R.K. Mishra, A classification of dark matter candidates with primarily spin-dependent interactions with matter, arXiv:1003.1912 [INSPIRE].

[46] JLQCD collaboration, $\mathrm{H}$. Ohki et al., Nucleon strange quark content from $N_{f}=2+1$ lattice QCD with exact chiral symmetry, Phys. Rev. D 87 (2013) 034509 [arXiv:1208.4185] [INSPIRE].

[47] H.-Y. Cheng and C.-W. Chiang, Revisiting scalar and pseudoscalar couplings with nucleons, JHEP 07 (2012) 009 [arXiv: 1202.1292] [INSPIRE].

[48] G. Bélanger, F. Boudjema, A. Pukhov and A. Semenov, MicrOMEGAs 3 : a program for calculating dark matter observables, Comput. Phys. Commun. 185 (2014) 960 [arXiv: 1305.0237] [INSPIRE].

[49] LUX collaboration, D.S. Akerib et al., First results from the LUX dark matter experiment at the Sanford Underground Research Facility, Phys. Rev. Lett. 112 (2014) 091303 [arXiv: 1310.8214] [INSPIRE].

[50] LUX collaboration, M. Szydagis et al., A detailed look at the first results from the Large Underground Xenon (LUX) dark matter experiment, arXiv:1402.3731 [INSPIRE].

[51] XENON1T collaboration, E. Aprile, The XENON1T dark matter search experiment, Springer Proc. Phys. 148 (2013) 93 [arXiv:1206.6288] [INSPIRE].

[52] M.A. Fedderke, E.W. Kolb, T. Lin and L.-T. Wang, Gamma-ray constraints on dark-matter annihilation to electroweak gauge and Higgs bosons, JCAP 01 (2014) 001 [arXiv:1310.6047] [INSPIRE].

[53] S. Dawson et al., Working group report: Higgs boson, arXiv:1310.8361 [INSPIRE].

[54] CMS collaboration, Search for an invisible Higgs boson, CMS-PAS-HIG-13-013, CERN, Geneva Switzerland (2013).

[55] CMS collaboration, Search for invisible Higgs produced in association with a $Z$ boson, CMS-PAS-HIG-13-018, CERN, Geneva Switzerland (2013).

[56] ATLAS collaboration, Search for invisible decays of a Higgs boson produced in association with a Z boson in ATLAS, Phys. Rev. Lett. 112 (2014) 201802 [arXiv:1402.3244] [INSPIRE].

[57] ATLAS collaboration, Updated coupling measurements of the Higgs boson with the ATLAS detector using up to $25 \mathrm{fb}^{-1}$ of proton-proton collision data, ATLAS-CONF-2014-009, CERN, Geneva Switzerland (2014). 
[58] G. Bélanger, B. Dumont, U. Ellwanger, J.F. Gunion and S. Kraml, Global fit to Higgs signal strengths and couplings and implications for extended Higgs sectors, Phys. Rev. D 88 (2013) 075008 [arXiv: 1306 .2941] [INSPIRE].

[59] S. Bhattacharya et al., Prospects for a heavy vector-like charge $2 / 3$ quark $T$ search at the LHC with $\sqrt{s}=14 \mathrm{TeV}$ and $33 \mathrm{TeV}$. "A Snowmass 2013 whitepaper", arXiv:1309.0026 [INSPIRE].

[60] Top Quark Working Group collaboration, K. Agashe et al., Working group report: top quark, arXiv:1311.2028 [INSPIRE].

[61] H.-C. Cheng, B.A. Dobrescu and J. Gu, Higgs mass from compositeness at a multi-TeV scale, JHEP 08 (2014) 095 [arXiv:1311.5928] [INSPIRE].

[62] H.S. Fukano, M. Kurachi, S. Matsuzaki and K. Yamawaki, Higgs as a top-mode pseudo, arXiv:1311.6629 [INSPIRE]. 